\title{
Identification of three new isolates of Tomato spotted wilt virus from different hosts in China: molecular diversity, phylogenetic and recombination analyses
}

Zhenjia Zhang ${ }^{1}$, Deya Wang ${ }^{1}$, Chengming Yu' ${ }^{1}$ Zenghui Wang ${ }^{1}$, Jiahong Dong ${ }^{2}$, Kerong Shi ${ }^{3^{*}}$ and Xuefeng Yuan ${ }^{1^{*}}$

\begin{abstract}
Background: Destructive diseases caused by Tomato spotted wilt virus (TSWV) have been reported associated with many important plants worldwide. Recently, TSWV was reported to infect different hosts in China. It is of value to clone TSWV isolates from different hosts and examine diversity and evolution among different TSW isolates in China as well as worldwide.

Methods: RT-PCR was used to clone the full-length genome ( $L, M$ and $S$ segments) of three new isolates of TSWV that infected different hosts (tobacco, red pepper and green pepper) in China. Identity of nucleotide and amino acid sequences among TSW isolates were analyzed by DNAMAN. MEGA 5.0 was used to construct phylogenetic trees. RDP4 was used to detect recombination events during evolution of these isolates.

Results: Whole-genome sequences of three new TSWV isolates in China were determined. Together with other available isolates, 29 RNA L, 62 RNA M and 66 RNA S of TSWV isolates were analyzed for molecular diversity, phylogenetic and recombination events. This analysis revealed that the entire TSWV genome, especially the $M$ and S RNAs, had major variations in genomic size that mainly involve the A-U rich intergenic region (IGR). Phylogenetic analyses on TSWV isolates worldwide revealed evidence for frequent reassortments in the evolution of tripartite negative-sense RNA genome. Significant numbers of recombination events with apparent $5^{\prime}$ regional preference were detected among TSWV isolates worldwide. Moreover, TSWV isolates with similar recombination events usually had closer relationships in phylogenetic trees.

Conclusions: All five Chinese TSWV isolates including three TSWV isolates of this study and previously reported two isolates can be divided into two groups with different origins based on molecular diversity and phylogenetic analysis. During their evolution, both reassortment and recombination played roles. These results suggest that recombination could be an important mechanism in the evolution of multipartite RNA viruses, even negative-sense RNA viruses.
\end{abstract}

Keywords: Tomato spotted wilt virus, Phylogenetic analysis, Reassortment, Recombination

\footnotetext{
* Correspondence: krshi@sdau.edu.cn; snowpeak77@163.com

${ }^{3}$ College of Animal Science and Technology, Shandong Agricultural University, Tai'an 271018, P. R. China

${ }^{1}$ College of Plant Protection, Shandong Agricultural University, No 61,

Daizong Street, Tai'an 271018, Shandong Province, P. R. China

Full list of author information is available at the end of the article
} 


\section{Background}

Tomato spotted wilt disease caused by Tomato spotted wilt virus (TSWV) was first described in 1919 in Australia (Brittlebank, 1919) [1] and has gradually spread to Europe, Africa, North America, and South America [2-11]. Recently, TSWV was detected in middle-eastern and fareastern countries in Asia including Iran, Japan, South Korea and China [12-17]. TSWV is mainly transmitted in a persistent manner by several species of thrips, especially the western flower thrip Frankliniella occidentalis, which may contribute to the worldwide spread of TSWV as well as to tomato spotted wilt disease [14, 18, 19]. TSWV infectes many important economic plants including tomato and potato, and causes serious damage including ringspot, black streak and tip dieback [20-22].

TSWV is the type member of the genus Tospovirus in the family Bunyaviridae [23]. TSWV has three singlestranded RNA segments denoted as L (8.9 kb), M (4.8 kb) and $\mathrm{S}(2.9 \mathrm{~kb})$, which together encode five proteins (Fig. 1). RNA L is a negative-sense RNA, whose complementary strand encodes the RNA-dependent RNA polymerase (RdRp), which is required for viral replication [24]. RNA $\mathrm{M}$ and $\mathrm{S}$ are ambisense with two ORFs, one expressed from the viral sense and the other the viral complementary strand. M plus-sense [M(+)RNA] encodes a nonstructural protein (NSm) responsible for cell-to-cell movement and its complementary minus-strand [M(-)RNA] encodes a Gn-Gc glycoprotein [25]. S(+)RNA encodes a second nonstructural protein (NSs) involved in the suppression of gene silencing and $\mathrm{S}(-)$ RNA encodes the nucleocapsid $(\mathrm{N})$ protein [26].

To date, whole-genome sequences have been determined for 19 TSWV isolates including 14 from South Korea, two from China (YN and CG-1), 1 from Brazil (BR-01) and 2 from Italy (p202/3WT and p105) (Additional file 1: Table S1) $[16,17,24]$. There are currently 26 full-length L, 59 full-length $M$, and 63 full-length $\mathrm{S}$ sequences deposited in the database (Additional file 1: Table S1). Previous studies suggested that both reassortment and recombination have contributed to the molecular diversity and evolution of TSWV based on partial sequences or regional wholegenome isolates $[17,27,28]$. However, only a few recombination events associated with TSWV have been described [17].
To identify the origin and evolution of TSWV in China, whole-genome sequences of three new TSWV isolates from tobacco, red pepper and green pepper in China were determined in this study. Molecular diversity and phylogenetic analysis revealed that all five Chinese TSWV isolates can be divided into two groups. Reassortment and huge numbers of recombination events were found based on phylogenetic and recombination analysis among all TSWV isolates worldwide.

\section{Results}

Sequencing of three new full-length TSWV isolates and examination of molecular diversity of TSWV in China

Three new TSWV isolates (YNta, YNrp and YNgp) originating in three different infected plants (tobacco, red pepper and green pepper) were obtained in Yunnan province (China) in 2013. The genome information of these isolates (YNta, YNrp and YNgp) is listed in Additional file 1: Table S1, which also contains information on previously reported TSWV isolates. RNA L of YNta, YNrp and YNgp were identical lengths (8913 nts), while the genomic sizes of RNAs $M$ and $S$ varied slightly due to size variation in the intergenic region (IGR) (Additional file 1: Table S1).

When two other previously reported Chinese TSWV isolates (YN and CG-1) were included in the analysis, new characteristic of TSWV were observed. In addition to size variations in IGR of the RNA $M$ and $S$, there were size variations in open reading frames (ORFs) and $5^{\prime}$ and $3^{\prime}$ untranslated regions (UTRs). For RNA L, the ORF in isolate CL4 (YN) is 8637 nt, compared with 8640 nt for other Chinese isolates. The 5'UTR and 3' UTR of isolate CL5 (CG-1) are 34 nt and $243 \mathrm{nt}$, respectively, compared with 33 nt and 240 nt in other Chinese isolates. These size variations contributed to the size differences among different isolates (CL4, $8910 \mathrm{nt}$; CL5, 8917 nt; CL1, CL2, CL3, 8913 nt). For RNA S, the 5 'UTR of CS5 is 86 nt compared with 88 nt for other Chinese isolates. In addition, the size of the IGR in CS5 (503 nt) is shorter than the IGR in other Chinese isolates (548 to $551 \mathrm{nt)}$ (Additional file 1: Table S1). Based solely on size similarities, TSWV isolates YNta, YNrp and YNgp may be more related than YN and CG-1.

In addition to genome size that reflects differences in ORFs and UTRs, nucleotide and amino acid sequences

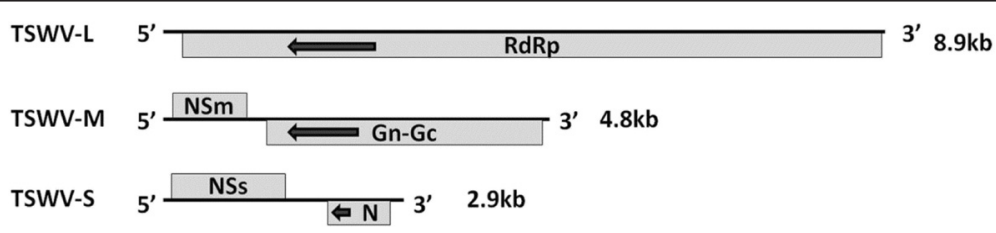

Fig. 1 Genomic organization of Tomato spotted wilt virus. RdRp: RNA-dependent RNA polymerase; NSm: nonstructural protein encoded by RNA M; Gn-Gc: Gn-Gc glycoprotein; NSs: nonstructural protein encoded by RNA S; N: nucleocapsid protein. Left pointing arrows denote that the ORF is on the viral complementary strand 
for the five Chinese TSWV isolates were also compared (Table 1). RNA L sequences were highly conserved, with nucleotide identity ranging from 99.17 to $99.52 \%$, whereas RNA S sequences were more variable (95.59 to $99.76 \%)$. For amino acid sequences, the $\mathrm{Gn}-\mathrm{Gc}$ protein was the most conserved (98.77 to $99.65 \%$ ), whereas NSs was more variable ( 97.05 to $99.79 \%$; Table 1). Among the five Chinese isolates, TSWV CG-1 was the most divergent (Table 1).

\section{Molecular diversity and phylogenetic relationships among TSWV isolates worldwide}

Whereas the TSWV Chinese isolates had slight differences in genome lengths when compared with each other, greater size differences were observed when all TSWV isolates were analyzed (Table 2). RNA L ranged from 8897 to $8917 \mathrm{nt}$, RNA M ranged from 4752 to $4830 \mathrm{nt}$, and RNA S ranged from 2916 to $3364 \mathrm{nt}$ (Table 2). The size of ORFs of NSm, Gn-Gc and N were stable, whereas NSs and the RdRp ORFs varied by $204 \mathrm{nt}$ and $15 \mathrm{nt}$, respectively. The IGRs in RNA M and S were highly variable in size, differing by 78 and $443 \mathrm{nt}$, respectively, whereas the 5'UTRs and 3'UTRs only had slight size variations (Table 2).

Phylogenetic trees based on the nucleotide sequences of RNA L, M and S were constructed. In the phylogenetic
Table 2 Molecular diversity of RNA L, M and S for TSWV isolates worldwide

\begin{tabular}{llll}
\hline & RNA L & RNA M & RNA S \\
\hline Genome size & $8897-8917(20)$ & $4752-4830(78)$ & 2916-3364 (448) \\
ORF1 & $8628-8643(15)$ & $909(0)$ & $1200-1404(204)$ \\
ORF2 & None & $3408(0)$ & $777(0)$ \\
5'UTR & $33-34(1)$ & $99-100(1)$ & $86-90(4)$ \\
3'UTR & $236-243(7)$ & $84-98(14)$ & $151-153(2)$ \\
IGR & None & $251-329(78)$ & $500-943(443)$
\end{tabular}

Values in brackets indicate the range of size variations (in nucleotides). ORF1 encodes the RdRp in RNA L, NSm is encoded by RNA M and NSs is encoded by RNA S. ORF2 encodes Gn-Gc in RNA M and N in RNA S. BD172070 (RNA L), HQ839730 (RNA S) and DQ431238 (RNA S) were not included in this analysis (Additional file 1: Table S1)

tree of RNA L, 29 isolates were divided into four clades (Fig. 2a). The first clade contained two sub-clades, one of which included a single Chinese isolate (CL4). The second sub-clade contained 21 isolates, inlcuding 14 from South Korea, three from China, one from Japan, two from Italy and 1 from the USA. The second clade contained only two isolates; KL20 from South Korea and UL3 from the USA (Fig. 2a). The third clade contained four isolates; two from Brail and two from the USA. The fourth clade contained a single Chinese isolate (CL5). The tree denoted that the evolutionary relationship and origin of the five

Table 1 Nucleotide and amino acid sequence identity for TSW isolates in China

\begin{tabular}{|c|c|c|c|c|c|c|}
\hline RNA L & CL1 & CL2 & CL3 & $C L 4$ & CL5 & \\
\hline CL1 & & 99.52 & 99.44 & 99.35 & 99.17 & \\
\hline $\mathrm{CL} 2$ & 99.17 & & 99.46 & 99.35 & 99.20 & \\
\hline CL3 & 99.13 & 99.20 & & 99.37 & 99.29 & \\
\hline$C L 4$ & 97.43 & 97.53 & 97.46 & & 99.18 & \\
\hline CL5 & 97.46 & 97.60 & 97.60 & 97.64 & & \\
\hline RNA M & CM1 & $\mathrm{CM} 2$ & $\mathrm{CM} 3$ & CM4 & CM5 & \\
\hline CM1 & & 99.29 & 99.41 & 99.62 & 97.97 & \\
\hline $\mathrm{CM} 2$ & $99.34(98.77)$ & & 99.29 & 99.31 & 97.74 & \\
\hline $\mathrm{CM} 3$ & $99.34(99.21)$ & $99.34(98.85)$ & & 99.43 & 97.84 & \\
\hline CM4 & $99.34(99.65)$ & $99.34(98.77)$ & $99.34(99.21)$ & & 97.99 & \\
\hline CM5 & $98.01(99.56)$ & $98.01(98.85)$ & $98.01(99.30)$ & 98.01(99.56) & & \\
\hline RNA S & $\mathrm{CS} 1$ & $\mathrm{CS} 2$ & $\mathrm{CS} 3$ & CS4 & CS5 & CS6 \\
\hline CS1 & & 99.43 & 99.56 & 99.43 & 95.63 & 99.56 \\
\hline CS2 & $98.72(98.84)$ & & 99.39 & 99.26 & 95.73 & 99.39 \\
\hline CS3 & $98.93(99.22)$ & 98.93(99.61) & & 99.56 & 95.80 & 99.76 \\
\hline CS4 & $98.93(98.06)$ & 98.93(98.45) & $99.14(98.84)$ & & 95.59 & 99.60 \\
\hline CS5 & $97.00(98.45)$ & $97.00(98.84)$ & $97.64(99.22)$ & $97.22(98.84)$ & & 95.86 \\
\hline CS6 & $99.14(98.84)$ & $99.14(99.22)$ & $99.79(99.61)$ & $99.36(99.22)$ & $97.86(99.61)$ & \\
\hline
\end{tabular}

CL1, CM1 and CS1 are the RNAs of TSWV isolate YNta; CL2, CM2 and CS2 are RNAs of TSWV isolate YNrp; CL3, CM3 and CS3 are RNAs of TSWV isolate YNgp; CL4, CM4 and CS4 are RNAs of TSWV isolate YN; CL5, CM5 and CS5 are RNAs of TSWV isolate CG-1; CS6 is another RNA of TSWV isolate KM-T

Percent identities were calculated using DNAMAN. Values above the diagonal shaded frames indicate the percentage of nucleotide sequence identity for $L, M$ and $S$ RNA, respectively. Values below the diagonal shaded frames indicate the percentage of amino acid sequence identity for RdRp, NSm (Gn-Gc) and NSs (N) encoded by $L, M$ and $S$, respectively 


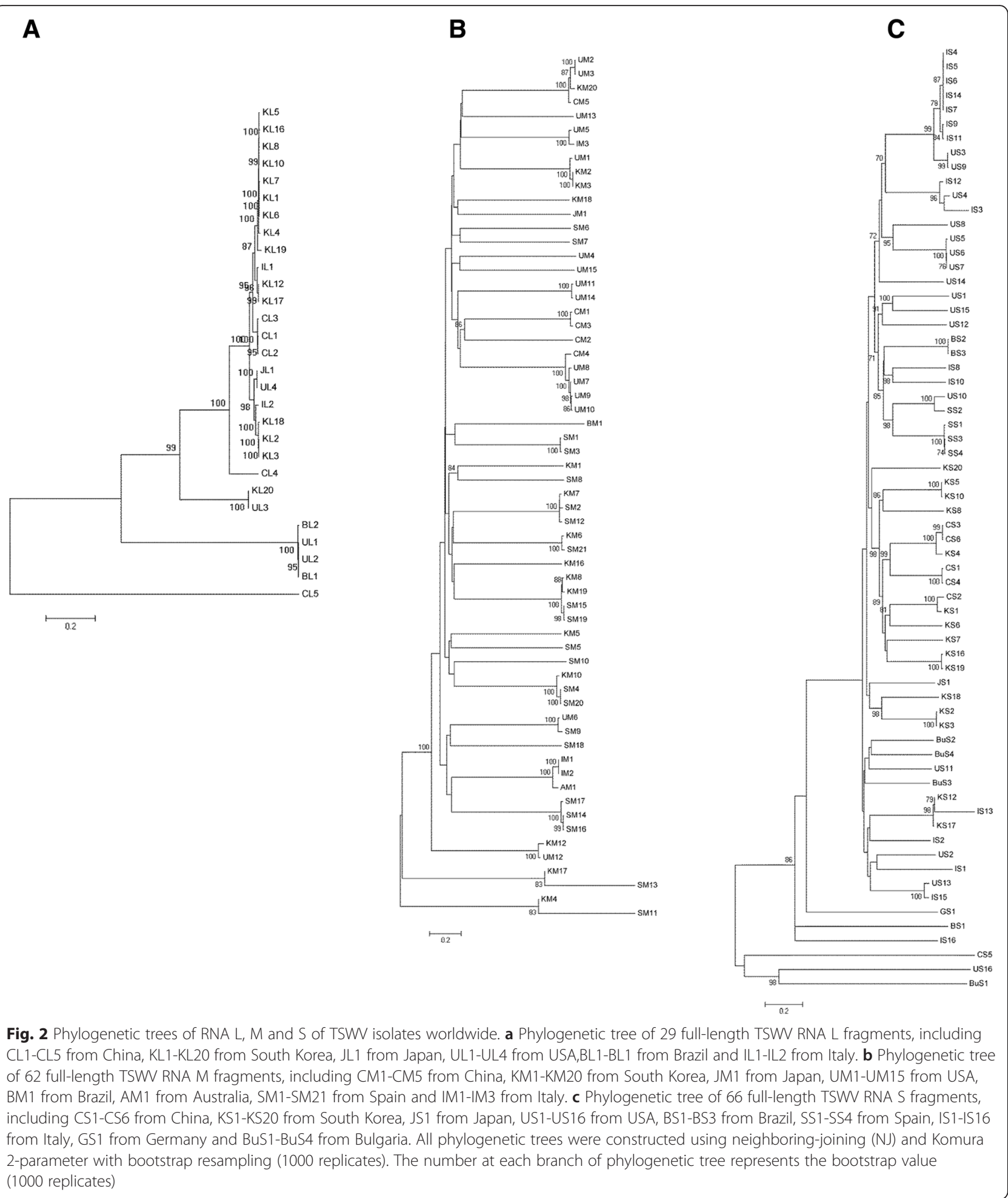

Chinese isolates were divergent. CL1, CL2 and CL3 formed one branch, and were most closely related to most of the South Korean isolates. CL4 was in one clade and CL5 was the most divergent, since it formed a clade that was independent from the other 28 isolates (Fig. 2a).
In the phylogenetic tree of RNA M, 62 isolates were divided into three clades (Fig. 2b). The first clade included two sub-clades, one of which contained 56 isolates from South Korea, China, Japan, Italy, Spain, and the USA; the second sub-clade included two isolates 
from South Korea and the USA. Both the second and third clades contained isolates from South Korea and Spain (Fig. 2b). M RNAs of the five Chinese isolates were more related than their RNA L since they all belonged to the first sub-clade of the first clade. CM1, CM2 and CM3, which also form a branch, have the closest relationship with Chinese isolate $\mathrm{CM} 4$ and four isolates from the USA (UM7, UM8, UM9 and UM10). CM5 is most closely related to one isolate from South Korea (KM20) and two isolates from the USA (UM2 and UM3) (Fig. 2b). Therefore, Chinese isolate RNA M were most closely related to some TSWV isolates from USA.

In the phylogenetic tree of RNA S, 66 isolates were divided into two clades (Fig. 2c). The first clade included four sub-clades, the first one of which included 60 isolates from South Korea, China, Japan, Italy, Bulgaria, Brazil and USA; the second, third and fourth sub-clade contained one isolate from Germany (GS1), Brazil (BS1) and Italy (IS16) respectively. The second clade contained two sub-clades, one of which contained a single Chinese isolate (CS5), and the second contained one isolate from USA (US16) and one isolate from Bulgaria (BuS1) (Fig. 2c). The origin of RNA $S$ of the 5 Chinese isolates differed from that of RNA L and M, with CS1, CS3 and CS4 comprising a branch with Chinese isolate CS6 and one isolate from South Korea (KS4). CS2 was most related to two isolates from South Korea (KS1 and KS6). CS5 formed a separate sub-clade in the second group (Fig. 2c). Therefore, with the exception of CS5, RNA S of Chinese isolates were most closely related to some TSWV isolates from South Korea.

Based on these phylogenetic trees, L, M and S of individual isolates could have different origins, implying the occurrence of frequent reassortment during the evolution of TSWV isolates. Among the Chinese isolates, RNA L (CL5) and S (CS5) of CG-1 were located in separate clades from the other four isolates, which correlated with the identity analysis of nucleotide sequence (Table 1). The three new isolates sequenced in this study (YNta, YNrp and YNgp) were more closely related to each other since their RNAs were contained in single branches in the phylogenetic trees of RNA L, M and S (Fig. 2a, b and c). These three new isolates also have a close relationship with isolate $\mathrm{YN}$ since they belonged to the same branch in phylogenetic trees of RNA M and S (Fig. 2b and c).

\section{Recombination analysis of TSWV isolates worldwide}

As described above, the phylogenetic trees of RNA L, M and $\mathrm{S}$ of TSWVs suggest the occurrence of frequent reassortment during the evolution of TSWV. To further define possible mechanisms of TSWV evolution, RNA recombination was examined using the program RDP4. Surprisingly, sufficient numbers of recombination events were detected so that only events supported by at least three methods with P-values $<1 \times 10^{-6}$ were included in the analysis (Figs. 3, 4 and 5; Additional file 2: Tables S2, Additional file 3: Table S3 and Additional file 4: Table S4).

For 29 full-length RNA L fragments of TSWV, 88 recombination events were detected in 27 RNA L fragments (Additional file 2: Table S2). Only 2 TSWV RNA L (CL5 from China and BL2 from Brazil) did not have detectable recombination events. Recombination events in RNA L were located throughout the genome, although most were located in the $5^{\prime}$ half of the $\mathrm{RdRp}$ ORF (Fig. 3; Additional file 2: Table S2). Some short recombination events located in the 3' terminal region are associated with recombination events in the $5^{\prime}$ half of the RdRp ORF, such as those located at $8722-4138$ in CL3 (event 9), 8128-1379 in KL19 (event 15), 87462865 in JL1 (event 67), 8726-4162 in UL3 (event 77) etc. (Fig. 3 and Additional file 2: Table S2). In addition, separate recombination events that were located in the 3' half of the RdRp ORF were detected in some RNA L from Chinese, South Korean and Italian isolates, such as recombination events located at 4781-6936 in CL4 (event 16), 5155-5629 in KL12, KL17 and IL1 (event 44, 50 and 85), 5154-5634 in KL1, KL4, KL6, KL7, KL8, KL10, KL16 and KL19 (event 19, 26, 29, 32, 35, 38, 41, 47 and 59), 5154-7248, 6308-6716 and 6910-7248 in KL19 (event 60-62) (Fig. 3 and Additional file 2: Table S2). Interestingly, recombination events having same breakpoints with same minor and major parents were detected in different RNA L, e.g., recombination events located at positions 5155-5629 (events 44/50/85) and 8912-367 (events 42/49/86) found in KL12, KL17 and IL1 (Fig. 3 and Additional file 2: Table S2). Similar cases included events $69 / 73$ and $70 / 72$ in UL1 and UL2; events $68 / 71 / 80$;events $1 / 4 / 7 / 17 / 24 / 27 / 30 / 33 / 36 / 39 / 45$; events $2 / 5 / 18 / 25 / 28 / 31 / 34 / 37 / 40 / 46 / 54 / 84$; events $20 /$ 22/51/79; events 64/75; events 43/48; events $19 / 26 / 32 /$ 35/38/41/47/59; events 6/9 and events 21/23/52 (detailed information are listed in Additional file 2: Table S2).

For 62 full-length TSWV RNA M fragments worldwide, 143 recombination events were totally detected from 56 RNA M fragments (Additional file 3: Table S3). Only in 6 RNA Ms (CM3, KM3 and KM18, JM1, UM1, SM11), no recombination event was detected. Preeminently within TSWV RNA M, recombination events are located at $5^{\prime}$ one-third of genome with only one exception, which occurred at position of 7-2626 in SM13 (event 99) (Fig. 4; Fig. 4d). In addition, there are three recombination events (including event 21, 33 and 99) bridging two ORF-coding regions (Fig. $4 \mathrm{~b}$ and d). Similar to the recombination events of RNA L, many short recombination events of RNA M located at 3' terminal region are simultaneously connected with those at $5^{\prime}$ one-third region of genome, such as recombination at 


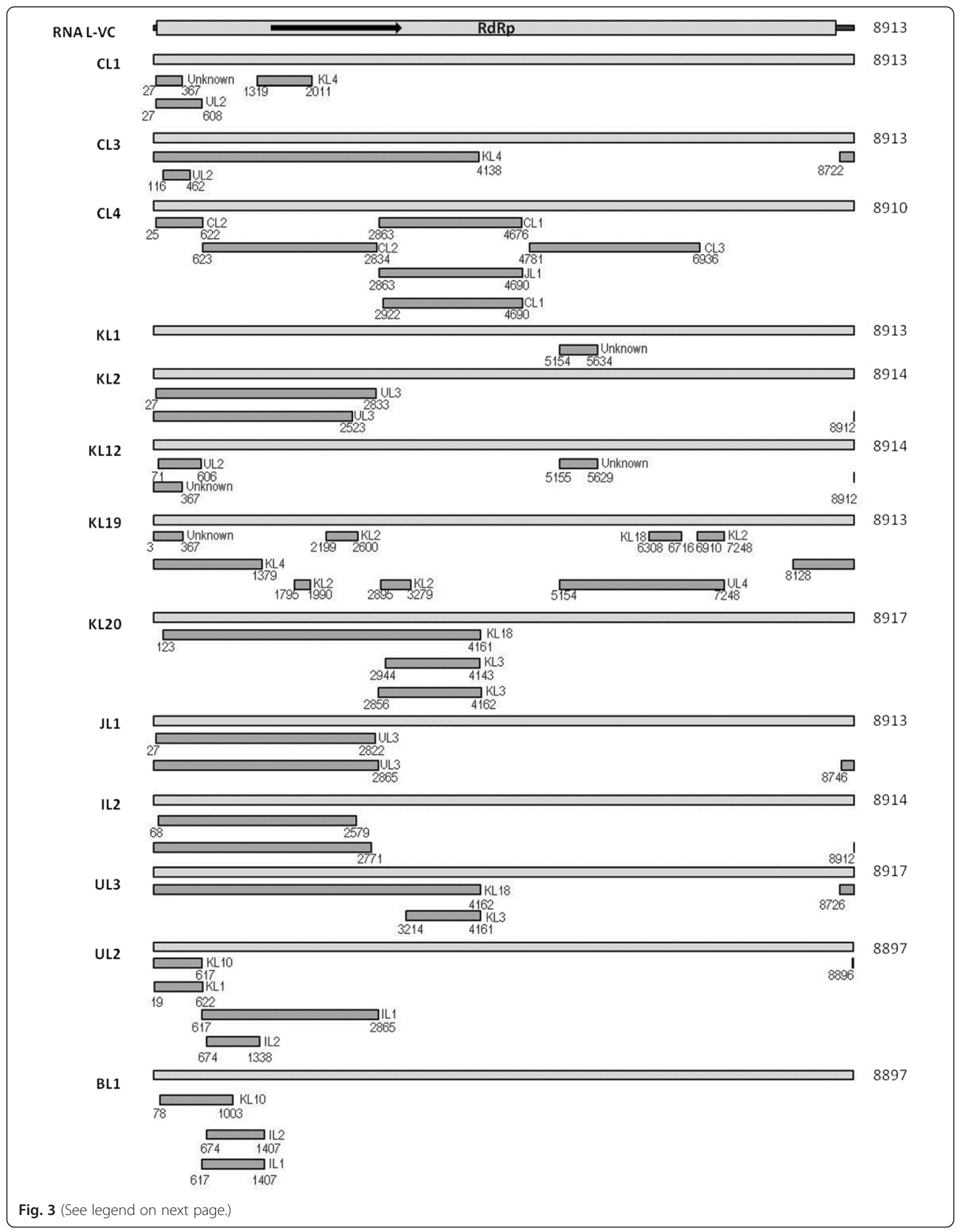


(See figure on previous page.)

Fig. 3 Analysis of putative recombination events in RNA L of TSWV isolates. Possible recombination events are indicated by black bars with minor parent and breakpoint positions of recombination sequences noted. Similar recombination events in different TSWV RNA L are shown once. Detailed information for each possible recombination event of TSW RNA L is provided in Additional file 2: Table S2. "VC" indicates viral complementary strand

4743-1110 and 4750-1125 in CM5 (event 8-9), 47421212 for UM3 (event 45), 4770-1115 for UM12 (event 66) and so on (Fig. 4; Additional file 3: Table S3). However, different from the recombination events of RNA L, no separate recombination event was detected at the 3 ' half of genomic RNA M. Recombination events with same breakpoints were also detected in different RNA M as the case of RNA L, for example, recombination located at position of 37-809 (events 133/138), 123-874 (events 134/139) and 974-1251(events 135/141) in IM1 and IM2 (Fig. 4 and Additional file 3: Table S3). Similar cases included events $82 / 122,83 / 123$ and $85 / 124$ in SM4 and SM20; events 107/111, 108/112 and 110/114 in SM16 and SM17; events 5/53; events 51/56/60; events 52/54/57/58; events 55/70; events $1 / 4$; events $101 / 109$; events $42 / 45 / 47$ / 142; events 137/143; events 105/119; events 6/59 (detailed information are listed in Additional file 3: Table S3).

For 66 full-length TSWV RNA S fragments, 174 recombination events were detected in 61 RNA $S$ (Additional file 4: Table S4). Only 5 TSWV RNA S (US16, BS2 and $\mathrm{BS} 3$, IS1, BuS1) did not have detectable recombination events. Similar to the recombination events of RNA M, recombination events in TSWV RNA $S$ were located within the 5' 2000 nt of the RNA with one exception: position 999-2833 in US8 (event 70) (Fig. 5; Fig. 5c; Additional file 4: Table S5). Also, many short recombination events located in $3^{\prime}$ terminal regions were similarly associated with these $5^{\prime}$ region recombination events, such as 28991772 in CS3 (event 5), 2908-1772 in CS6 (event 10), 2883-1760 in KS5 (event 21), 2813-1616 in KS16 (event 35), 2943-1652 in BuS3 (event 96), etc. (Fig. 5; Additional file 4: Table S4). Furthermore, as found in RNAs L and $\mathrm{M}$, recombination events with identical breakpoints were detected in different RNA S, e.g., recombination events located at position 78-1622, 1234-1761 and 27701784 in SS3 and SS4 (Fig. 5 and Additional file 4: Table S4). Similar cases included events 169/172, 167/171 and 166/170 in SS3 and SS4; events 109/115/121/126/154 and $104 / 110 / 117 / 123 / 149$ in IS4, IS5, IS6, IS7 and IS14; events 58/63; events 80/87; events 62/66/68; events 107 / 113; events $112 / 119$; events $33 / 37$; events $161 / 106 / 111$ / 118/124/132/141/150; events 108/120; events 116/122/ 155 ; events 84/91/93 (detailed information are listed in Additional file 4: Table S4).

To confirm the reliability of unusual frequent recombination events among TSWV in this study, phylogenetic trees based on recombination breakpoints were constructed to assess the relationship between the receptor and donor sequence. For RNA L, two phylogenetic trees based on fragment 1-2850 and 1-650 were constructed (Fig. 6a and b) since many recombination events have similar breaking points with these two regions (Fig. 3; Additional file 2: Table S2). For recombination among RNA L of TSWV, events 20/22/51/66/79 have similar breaking points (27-2833) in KL2, KL3, KL18, JL1 and UL4 (Additional file 2: Table S2), which were located in the same branch including their co-minor parent UL3, while their co-major parent KL16 belonged to the other further branch (Fig. 6a). It is suggested the intrinsic relationship between recombination events and phylogenetic trees, which confirmed the reliability of recombination events 20/22/51/66/79 (Fig. 6a; Additional file 2: Table S2). Events 2/5/18/25/28/31/37/40/46/54/84 have similar breaking points (27-606) with same minor and major parents (UL2/IL2) in CL1, CL2, KL1, KL4, KL5, KL6, KL8, KL10, KL16, KL19 and IL1 (Additional file 2: Table S2), which belonged to the same main branch including their cominor parent UL2, while their co-major IL2 formed into a separate clade (Fig. 6b). It also implied the intrinsic relationship between recombination and phylogenetic analysis. For RNA M, one phylogenetic tree based on the fragment 1-1250 was constructed (Fig. 6c). Data from this phylogenetic tree supported the recombination events $3 / 5 / 11 / 15 / 20$ / 30/41/60/67/69/76/87/92 having similar breaking points (Fig. 6c; Additional file 3: Table S3). For events 3 and 5, CM2, CM4 and their co-minor parent CM1 formed into a branch, while their major parent SM19 and UM8 belonged to other branches (Additional file 3: Table S3; Fig. 6c). For event 11, KM1 and its minor parent KM8 formed into a branch, while its major parent BM1 belonged to other main branch (Additional file 3: Table S3; Fig. 6c). In addition, the status about recombinant sequence and its minor parent having close relationship in the phylogenetic tree is also applied to events 15/20/30/41/60/67/69/76/87/92 (Additional file 3: Table S3; Fig. 6c). These data verified the reliability of recombinant events of RNA $M$ of TSWV. Conclusively, there are frequent recombinant events among TSWV.

\section{Discussion}

\section{Molecular diversity among 5 TSWV Chinese isolates}

International trade of seeds and vegetables, and the presence or importation of vector thrips have allowed for the rapid spread of TSWV and associated diseases worldwide [20, 21]. During this period of expansion, TSWV has been continuing to evolve, possibly depending on host and environmental factors. For the current 


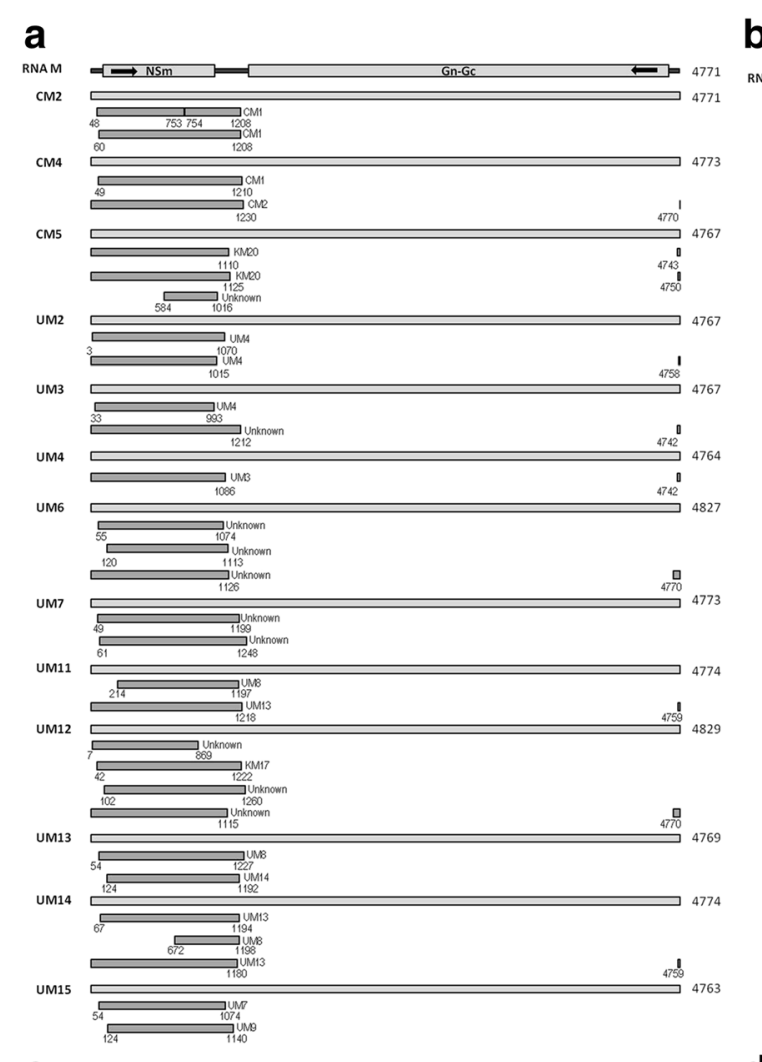

b

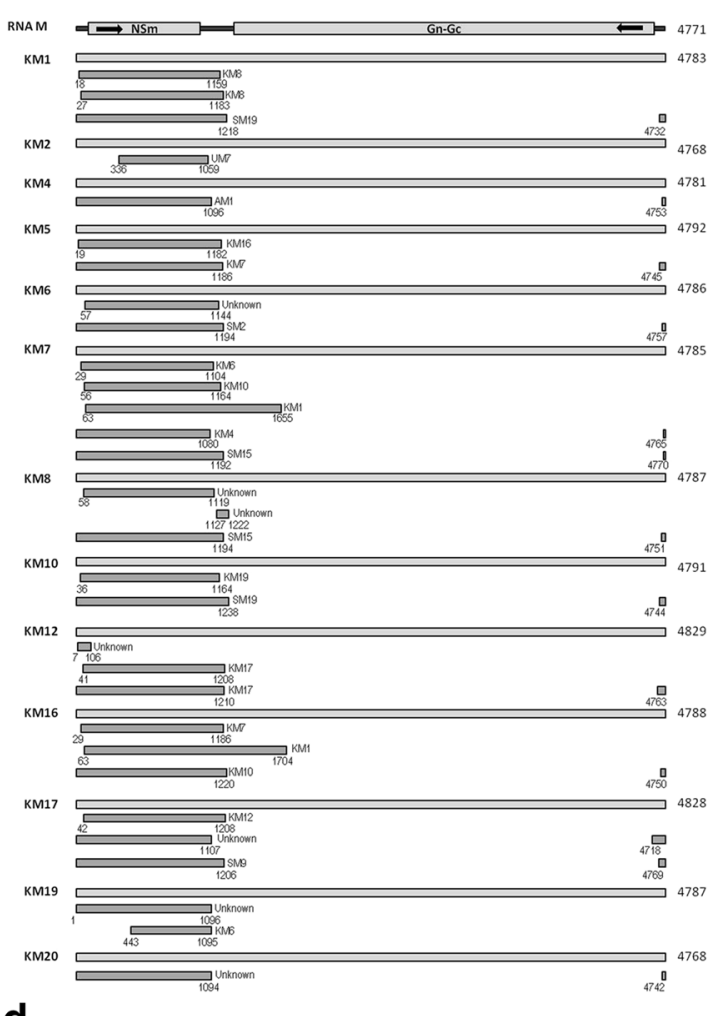

C

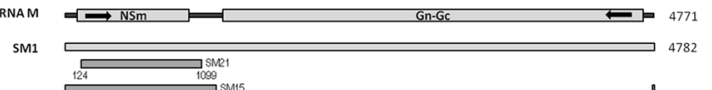

SM2

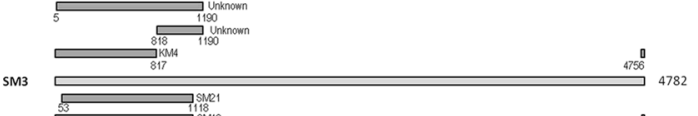

SM4
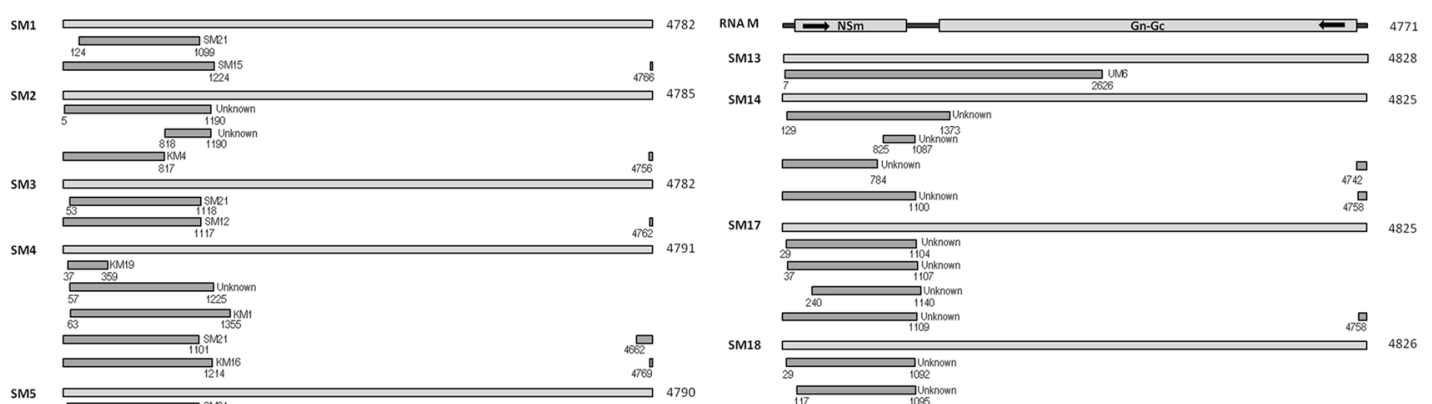

SM5

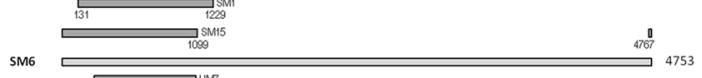

SM6
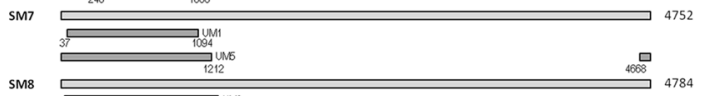

SM8

SM9

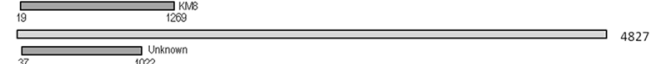

SM10 $\overbrace{1008}^{\text {SM10 }} 4830$
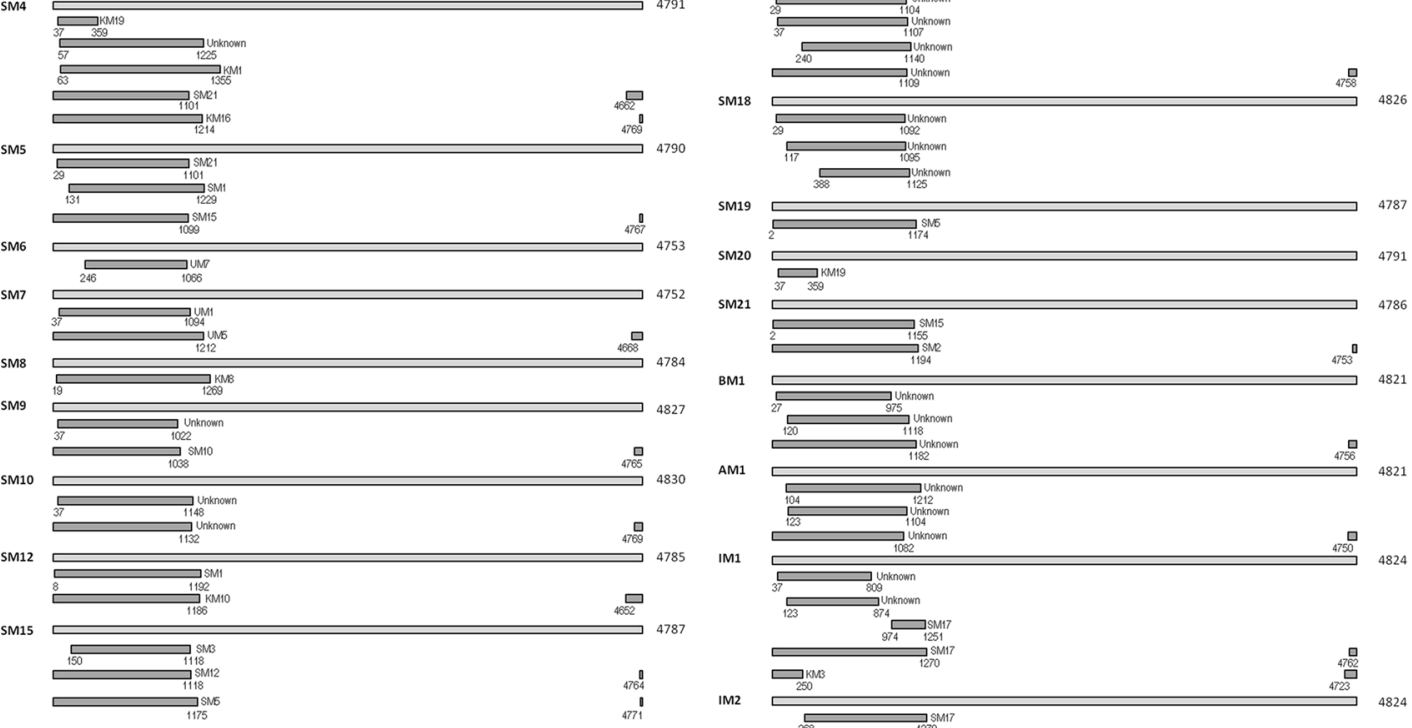

Fig. 4 Analysis of possible recombination in RNA M of TSWV isolates. This figure includes 4 separate figures (4a, $4 \mathbf{b}$, $4 \mathbf{c}$ and $4 \mathbf{d}$ ) due to the large amount of recombination events. Detailed information for each recombination event is provided in Additional file 3: Table S3. See legend to Fig. 3 for additional information 


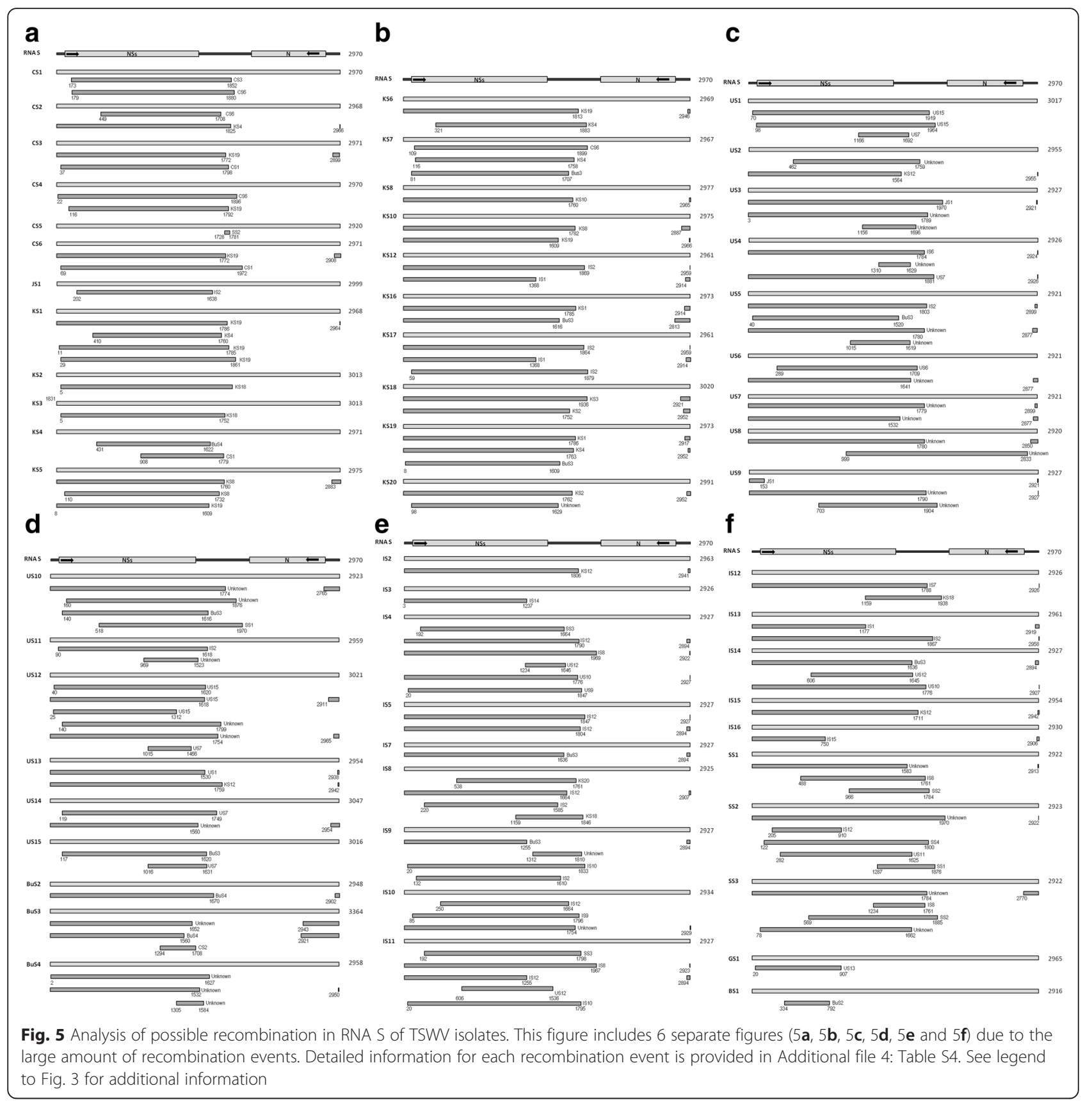

study, three new isolates of TSWV were cloned from different hosts (tobacco, red pepper and green pepper) grown in Chinese fields and then compared with two previously cloned TSWV isolates from tomato and lettuce in China [16]. Among these five TSWV isolates in China, only slight variations were found in the sizes of $\mathrm{L}, \mathrm{M}$ and S RNA (Additional file 1: Table S1). When 29 RNA L, 62 RNA $M$ and 66 RNA $S$ were compared (Additional file 1: Table S1), the range of variation was considerable but mainly in untranslated regions (Table 2). For the 5 ORFs encoded by the TSWV (-) strand or (+) strand, only RdRp and NSs vary in size (15 and $204 \mathrm{nt}$, respectively) due to mutations that introduce nonsense codons (Table 2). Currently, it is not known if these size changes affect infectivity of TSWV, or if the clones represent viable viral RNAs (there is no reverse genetic system available for TSWV). CG-1 (CL5, CM5 and CS5 RNAs), isolated from lettuce in China may be considered a new TSWV species based on molecular diversity (Table 1), and phylogenetic analyses (Fig. 2). In addition, the RdRp amino acid sequence of TSWV-YN from tomato in China differs from other 4 Chinese isolates (Table 1). In contrast, YNta, YNrp and YNgp are relative stable and are found together in one branch of the RNA L and M phylogenetic trees. 


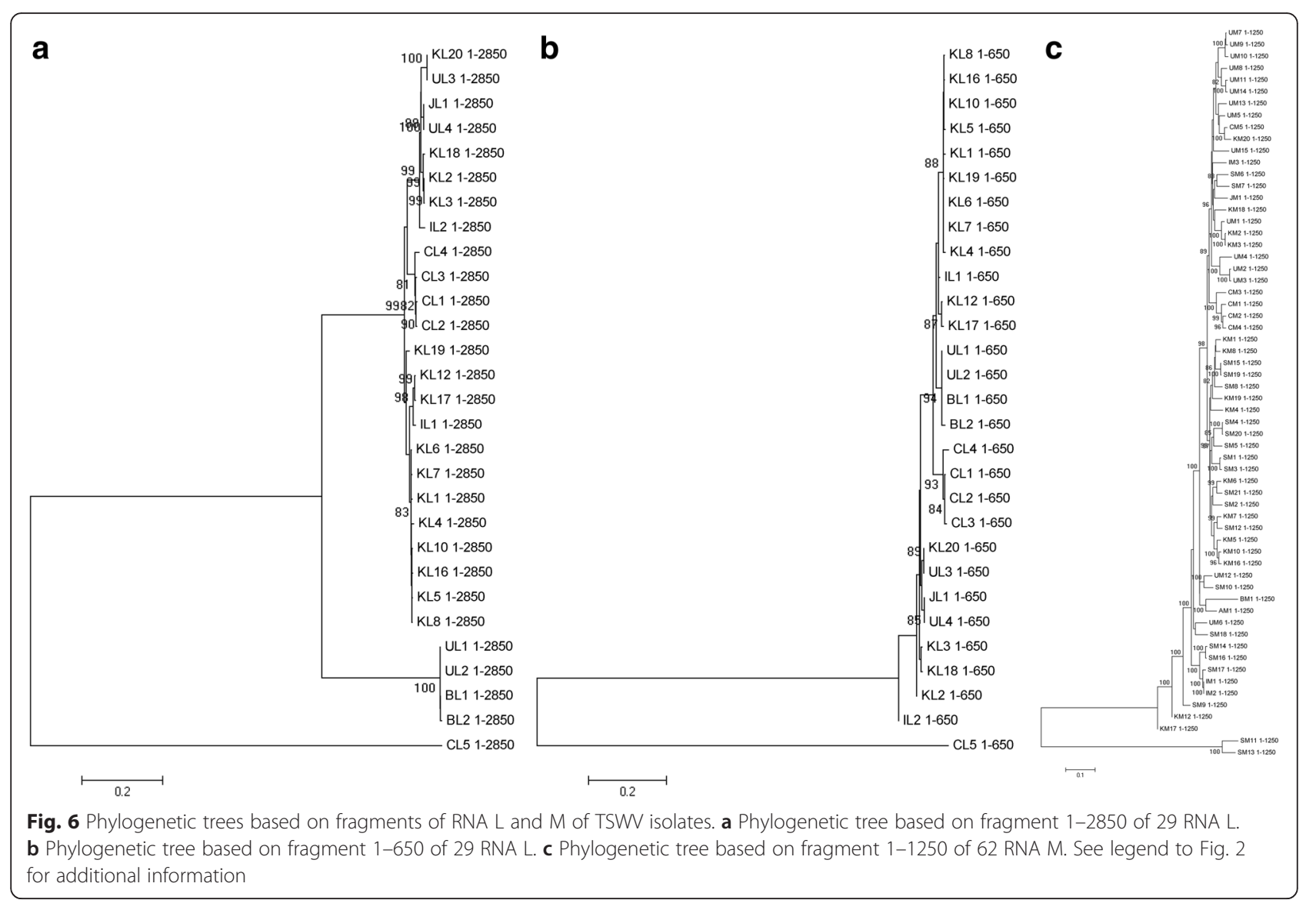

The phylogenetic trees revealed that the three RNAs of the 5 Chinese TSWV isolates do not group together in the same clades, suggesting that these viruses have undergone reassortment during their evolution, as has been reported to occur for TSWV $[17,28]$. RNA L of CL1, CL2, CL3 and CL4 are related to the RNA L of isolates from South Korea, while CL5 is a new species with an unclear origin (Fig. 2a). For RNA M, the origin of CM1, CM2, CM3, and CM4 is RNA M from the USA, while the origin of CM5 is RNA M from either the USA or South Korea (Fig. 2b). For RNA S, the origin of CS1, CS2, CS3 and CS4 is RNA S from South Korea, while the origin of CS5 is RNA S from either the USA or Bulgaria (Fig. 2c).

\section{High levels of recombination events have occurred among TSWV isolates worldwide}

Besides reassortment of fragments $\mathrm{L}, \mathrm{M}$ and $\mathrm{S}$ in different TSWV isolates, many recombination events were detectable in nearly all RNA L, M and $\mathrm{S}$ examined, differing from a previous report using fewer isolates that suggested only a low level of recombination events have occurred [17]. There are currently two major hypotheses concerning the contribution of recombination to the evolution of modern RNA viruses. One suggests that recombination is a major evolutionary mechanism for single-stranded RNA viruses and a minor mechanism for the evolution of multipartite RNA viruses [29, 30], which is supported by reports on Soybean mosaic virus and CMV [31-33]. The second hypothesis is that both recombination and reassortment are important evolutionary mechanisms for multipartite RNA viruses, which is supported by studies of Brome mosaic virus [34]. Our study provides additional support for the second hypothesis, although previous studies have shown that homologous recombination seems to be very rare or even absent in most negative-sense RNA viruses [35, 36].

For all three RNAs of TSWV isolates, recombination events were mainly detected in the $5^{\prime}$ half of the RNA. The reason for this preference is not clear. One possibility is that RdRp firstly bound RNA template, synthesized complementary strands and then had chance to facilitate recombination among different complementary strands. For bi-cistronic RNA $M$ and $S$, the vast majority of recombination spanned only the ORF located at $5^{\prime}$, suggesting that recombination preferred to occur at ORF-coding region instead of untranslated regions and different ORFs are undergoing separate evolution. In addition, recombination events involving short fragments in the $3^{\prime}$ terminal region were connected with $5^{\prime}$ 
region recombination events in all three RNAs, which may cause by closed structure of TSWV RNAs resulting from the long base-pairing between $5^{\prime}$ and $3^{\prime}$ terminal sequences (5'-GAGCAAUUGUGUCA------UGACACAA UUGCUCU-3') .

The similar recombination events in different RNA L, $\mathrm{M}$ and S of TSWV imply a connection between recombination and the phylogenetic tree. Isolates with similar or identical recombination breakpoints are grouped together. For example, for RNA L, three isolates (KL12, KL17, IL1) have recombination events located at positions 5155-5629 and 8912-367 (Additional file 2: Table S2, Fig. 3), forming a cluster in the phylogenetic tree of RNA L (Fig. 2a). The second characteristic is that isolates in which no recombination was detected are usually clustered in separate groups or branches. For example, CL5 in which no recombination was detected belonged to a separate group from the other 28 isolates (Fig. 2a).

\section{Conclusions}

Whole-genome sequences of three new TSWV isolates from different hosts in China were determined. Based on molecular diversity on 29 RNA L, 62 RNA M and 66 RNA S of TSWV, it is suggested that the entire TSWV genome, especially the $\mathrm{M}$ and $\mathrm{S}$ RNAs, have undergone extreme variations in genomic size that mainly involve the A-U rich intergenic region (IGR). Phylogenetic analyses on TSWV isolates worldwide revealed evidence for frequent reassortments. In addition, all five Chinese TSWV isolates can be divided into two groups with different origins based on molecular diversity and phylogenetic analysis. Significant numbers of recombination events were detected among TSWV isolates worldwide with apparent regional preference and showed inherent connection with phylogenetic trees. These results suggest that recombination could be an important mechanism in the evolution of multipartite RNA viruses.

\section{Methods \\ Whole-genome cloning and sequencing of three new TSWV isolates from China}

Tobacco, red pepper and green pepper plants with typical symptoms of TSWV infection were collected from Luxi county, Honghe city (Yunnan province) in China in 2013 (specific permission was not required). Three new TSWV isolates (YNta, YNrp and YNgp) were identified following cloning and sequencing. In brief, total RNA was extracted from leaves of putatively infected tobacco, red pepper or green pepper using TRIzol Reagent (TransGen). For cDNA synthesis, total RNA was combined with dNTPs and primers corresponding to the 3' ends of L, M or S RNAs (Additional file 5: Table S5). After 5 min incubation at $65{ }^{\circ} \mathrm{C}, 5$ units of PrimeScript reverse transcriptase and $5 \times$ buffer (Takara) was added and incubation continues at $42{ }^{\circ} \mathrm{C}$ for $1.5 \mathrm{~h}$. Following cDNA synthesis, PCR amplification was performed using LA Taq DNA polymerase and pairs of primers corresponding to internal and terminal regions of $\mathrm{L}, \mathrm{M}$ or $\mathrm{S}$ RNAs (Additional file 5: Table S5). Three fragments were amplified for L (positions 1-2722, 2512-5927 and 5906-8913), two fragments were amplified for $M$ (positions 1-3401 and 2215-4772), and full-length cDNA was amplified for S. All PCR products were cloned into pMD18-T (Takara) and sequenced using universal vector primers and specific primers designed for TSWV components (Additional file 5: Table S5). At least three clones for each PCR product were sequenced to avoid experimental errors.

\section{Sequence assembly, construction of phylogenetic trees and recombination analysis}

Sequence assembly was accomplished using DNAMAN, which was also used to analyze nucleotide and amino acids sequences among TSWV isolates. After sequence assembly, complete sequences of $\mathrm{L}, \mathrm{M}$ and $\mathrm{S}$ for the three new TSWV isolates were deposited into GenBank with accession numbers from KM657114 to KM657122 (http://www.ncbi.nlm.nih.gov/nucleotide/).

The phylogenetic tree was constructed using the MEGA 5.0 software package [37] based on methods of neighborjoining and Kimura 2-parameter. Bootstrap resampling (1000 replicates) was used to ensure reliability of individual nodes in the phylogenetic tree. Values lower than $70 \%$ were hidden. Searches for recombination events employed six methods including RDP, GENECONV, Bootscan, Maxchi, Chimaera and SiScan, implemented in RDP4 [38], with likely parental isolates and recombination break points determined using default settings. Recombination events were noted if supported by at least three different methods $\left(\right.$ P-values $\left.<1.0 \times 10^{-6}\right)$. Sequences of additional full-length TSWV isolates were obtained from http://www.ncbi.nlm. nih.gov/nucleotide/ (See Additional file 1: Table S1 for detailed information).

\section{Additional files}

Additional file 1: Table S1. TSWV isolates used in this study. TSWV isolates from different countries use different abbreviations as followed, China (CL, CM and CS), South Korea (KL, KM and KS), Japan (JL, JM, JS), USA (UL, UM, US), Brazil (BL, BM, BS), Italy (IL, IM IS), Australia (AM), Spain (SM, SS), Germany (GS), Bulgaria (BuS). CS: Complementary sequence; N/A: Not analyzed. (DOCX $35 \mathrm{~kb}$ )

Additional file 2: Table S2. Summary of recombination events in different full-length TSWV L isolates using RDP4. NS: not significant. (DOCX $24 \mathrm{~kb}$ )

Additional file 3: Table S3. Summary of recombination events in different full-length TSWV L isolates using RDP4. NS: not significant. (DOCX $31 \mathrm{~kb})$ 
Additional file 4: Table S4 Summary of recombination events in different full-length TSWV L isolates using RDP4. NS: not significant. (DOCX $33 \mathrm{~kb}$ )

Additional file 5: Table S5. Primers used in this study. Primers were designed based on previously reported TSW sequences. W: A/T, M: A/C, R: G/A, Y: C/T. (DOCX $15 \mathrm{~kb})$

\section{Competing interests}

The authors declare that they have no competing interests.

\section{Authors' contributions}

ZZ contributed to the design of the study, RT-PCR of new TSW isolates, data analysis and drafting the manuscript. DW contributed to detection of TSW and data analysis. CY contributed to data analysis. ZW contributed to data analysis. JD contributed to the sample collection. KS and XY contributed to the design of the study, data analysis and drafting the manuscript. All authors read and approved the final manuscript.

\section{Acknowledgements}

This work was supported by grants from The National Natural Science Foundation of China (31370179), and The Shandong Province Natural Sciences Foundation of China (ZR2013CM015). We are grateful to Prof. Anne E. Simon, University of Maryland (USA) for valuable suggestions and critical modification on the manuscript.

\section{Author details}

${ }^{1}$ College of Plant Protection, Shandong Agricultural University, No 61 Daizong Street, Tai'an 271018, Shandong Province, P. R. China. ${ }^{2}$ Resources Institute, Yunnan Academy of Agricultural Sciences, Yunnan Provincial Key Lab of Agricultural Biotechnology, Key Lab of Southwestern Crop Gene Resources and Germplasm Innovation, Ministry of Agriculture, Kunming 650223, China. ${ }^{3}$ College of Animal Science and Technology, Shandong Agricultural University, Tai'an 271018, P. R. China.

Received: 17 August 2015 Accepted: 21 December 2015

Published online: 14 January 2016

\section{References}

1. Brittlebank CC. Tomato diseases. J Agri Victoria. 1919;27:231-5.

2. Smith KM. Studies on plant virus diseases. XI. Further experiments with a ringspot virus: its identification with spotted wilt of tomato. Ann Appl Biol. 1932;19:305-20.

3. Moore ES. The kromnek or Kat river diease of tobacco and tomato in the east province (South Africa). Dept. Agric., union of South Africa. Sci Bull. 1933;123:5-28

4. Haliwell RS, Philley G. Spotted wilt of peanut in Texas. Plant Dis Rep. 1974; 58:23-5.

5. Culbreath AK, Csinos AS, Bertrand PF, Demski JW. Tomato spotted wilt virus epidemic in flue-cured tobacco in Georgia. Plant Dis. 1991;75:483-5.

6. Maluf WR, Toma-Brahini M, Corte RD. Progress in breeding tomatoes for resistance to tomato spotted wilt. Braz J Genet. 1991;14:509-25.

7. Peters D, Wijkamp I, van de Wetering F, Goldbach R. Vector relations in the transmission and epidemiology of tospoviruses. Acta Hort. 1996;431:29-43.

8. EPPO: Data sheets on quarantine pests: Tomato spotted wilt tospovirus. (revision of original 1997c data sheet). 2004

9. Abad JA, Moyer JW, Kennedy GG, Holmes GA, Cubeta MA. Tomato spotted wilt virus on potato in eastern North Carolina. Am J Potato Res. 2005;82:255-61.

10. Rosales M, Pappu HR, Arayam C, Aljaro A. Characterization of tomato spotted wilt virus (tospovirus, bunyaviridae) from lettuce (lactuca sativa) in Chile. Phytopathology. 2007;97:S101

11. Sivprasad BJ, Gubba A. Isolation and molecular characterization of Tomato spotted wilt virus (TSW) isolates occurring in South Africa. Afr J Agric Res. 2008:3:428-34.

12. Mumford RA, Barker I, Wood KR. The biology of the tospoviruses. Ann Appl Biol. 1996;128:159-83.

13. Golnaraghi AR, Shahraeen N, Pourrahim R, Ghorbani S, Farzadfar S. First report of Tomato spotted wilt virus on soybean in Iran. Plant Dis. 2001;85:1290.

14. Jones DR. Plant viruses transmitted by thrips. Eur J Plant Pathol. 2005;113:119-57.
15. Massumi H, Samei A, Hosseini Pour A, Shaabanian M, Rahimian H. Occurrence, distribution, and relative incidence of seven viruses infecting greenhouse-grown cucurbits in Iran. Plant Dis. 2007;91:159-63.

16. Hu Z, Feng Z, Zhang Z, Liu Y, Tao X. Complete genome sequence of a Tomato spotted wilt virus isolate from China and comparison to other TSW isolates of different geographic origin. Arch Virol. 2011;156:1905-8.

17. Lian S, Lee J-S, Cho WK, Yu J, Kim M-K, Choi H-S, et al. Phylogenetic and recombination analysis of tomato spotted wilt virus. PLoS One. 2013;8(5):e63380.

18. Rotenberg D, Jacobson AL, Schneweis DJ, Whitfield AE. Thrips transmission of tospoviruses. Curr. Opin. Virol. 2015;15:80-9.

19. Kirk W, Terry $L$. The spread of the western flower thrips Frankliniella occidentalis (Pergande). Agric For Entomol. 2003;5:301-10.

20. Prins $M$, Goldbach R. The emerging problem of tospovirus infection and nonconventional methods of control. Trends Microbiol. 1998;6:31-5.

21. Pappu $H_{\text {, Jones }}$, Jain $R$. Global status of tospovirus epidemics in diverse cropping systems: Successes achieved and challenges ahead. Virus Res. 2009;141:219-36.

22. Hanssen I, Lapidot M, Thomma B. Emerging viral diseases of tomato crops. Mol Plant Microbe Interact. 2010;23:539-48.

23. Fauquet CM, Mayo MA, Maniloff J, Desselberger U, Ball LA. Virus taxonomy. 8th report of the international committee on taxonomy of viruses. San Diego: Elsevier Academic Press; 2005.

24. de Haan P, Kormelink R, Oliveira R, Poelwijk F, Peters D, Goldbach R. Tomato spotted wilt virus L RNA encodes a putative RNA polymerase. J Gen Virol. 1991;72:2207-16.

25. Kormelink R, Haan P, Meurs C, Peters D, Goldbach R. The nucleotide sequence of the M RNA segment of Tomato spotted wilt virus, a bunyavirus with two ambisense RNA segments. J Gen Virol. 1992;73:2795-804.

26. de Haan P, Wagemakers L, Peters D, Goldbach R. The S RNA segment of Tomato spotted wilt virus has an ambisense character. J Gen Virol. 1990:71:1001-7.

27. Qiu WP, Geske SM, Hickey CM, Moyer J. Tomato spotted wilt Tospovirus genome reassortment and genome segment-specific adaptation. Virology. 1998;244(1):186-94

28. Tentchev D, Verdin E, Marchal C, Jacquet M, Aguilar J, Moury B. Evolution and structure of Tomato spotted wilt virus populations: evidence of extensive reassortment and insights into emergence processes. J Gen Virol. 2011;92:961-73

29. Chao L. Evolution of sex in RNA viruses. J Theor Biol. 1988;133:99-112.

30. Pressing J, Reanney D. Divided genomes and intrinsic noise. J Mol Evol. 1984:20:135-46.

31. Fraile A, Alonso-Prados JL, Aranda MA, Bernal JJ, Malpica JM, Garcia-Arenal F. Genetic exchange by recombination or reassortment is infrequent in natural populations of a tripartite RNA plant virus. J Virol. 1997:71:934-40.

32. Gagarinova A, Babu M, Stro "mvik M, Wang A. Recombination analysis of Soybean mosaic virus sequences reveals evidence of RNA recombination between distinct pathotypes. Virol J. 2008;5:143.

33. Seo J, Ohshima K, Lee H, Son M, Choi H, Lee HS, et al. Molecular variability and genetic structure of the population of Soybean mosaic virus based on the analysis of complete genome sequences. Virology. 2009:393:91-103.

34. Bruyere A, Wantroba M, Flasinski S, Dzianott A, Bujarski J. Frequent homologous recombination events between molecules of one RNA component in a multipartite RNA virus. J Virol. 2000;74:4214-9.

35. Chare ER, Gould EA, Holmes EC. Phylogenetic analysis reveals a low rate of homologous recombination in negative-sense RNA viruses. J Gen Virol. 2003;84:2691-703

36. Han GZ, Worobey M. Homologous recombination in negative sense RNA viruses. Viruses. 2011;3(8):1358-73.

37. Tamura K, Peterson D, Peterson N, Stecher G, Nei M, Kumar S. MEGA5: molecular evolutionary genetics analysis using maximum likelihood, evolutionary distance, and maximum parsimony methods. Mol Biol Evol. 2011:28:2731-9.

38. Martin DP, Lemey P, Lott M, Moulton V, Posada D, Lefeuvre P. RDP3: A flexible and fast computer program for analyzing recombination. Bioinformatics. 2010;26:2462-3. 